\title{
THE EXISTENCE OF LINE INVOLUTIONS OF ORDER GREATER THAN THREE POSSESSING A LINEAR COMPLEX OF INVARIANT LINES
}

\author{
C. R. WYLIE, JR.
}

Introduction. In a recent paper [1] attention was called to a new family of line involutions in $S_{3}$ furnishing examples of involutions of all orders, $m, \geqq 4$ with complexes of invariant lines of all possible orders, $i$, from 2 up to the maximum, $[(m+1) / 2]$. Since involutions of all orders without a complex of invariant lines are known to exist, and since examples of all possible involutions of order $<4$ are known, the only involutions for which existence examples remain to be supplied are those whose orders are $\geqq 4$ and whose invariant lines form a linear complex. It is the purpose of this note to define a class of involutions having these properties, thus establishing the existence of line involutions corresponding to every admissible set of characteristics $(m, n, i, k)[1]$. In our development we shall work exclusively on the nonsingular $V_{4}^{2}$ in $S_{6}$ into whose points the lines of $S_{3}$ are mapped in a 1:1 way by the well known interpretation of the Plücker coordinates of a line in $S_{3}$ as point coordinates in $S_{5}$.

1. The definition of the involution. On $V_{4}^{2}$ let there be given a point $O$ and a plane $\pi$ in general position, and let $\lambda$ be the line in which the tangent hyperplane to $V_{4}^{2}$ at $O$ meets $\pi$. Moreover, let $C_{\alpha}$ be a curve of order $\alpha$ lying on $V_{4}^{2}$ and meeting $\pi$ in $\alpha-1$ points, $\beta$ of which fall on the line $\lambda$. Obviously $C_{\alpha}$ lies in an $S_{4}$ and is rational. Finally let $\Gamma$ be the set of points on $V_{4}^{2}$ which represents a general linear complex of lines in $S_{3}$; in other words, $\Gamma$ is the intersection of $V_{4}^{2}$ and a general $S_{4}$.

Now if $P$ is a general point of $V_{4}^{2}$ (the image of a general line in $S_{3}$ ), then $P O \pi$ is a 4 -space which meets $C_{\alpha}$ in the $\alpha-1$ fixed points common to $C_{\alpha}$ and $\pi$, and in one additional point $Q$ which varies with $P$. The point $Q$ will of course coincide with one of the fixed intersections of $C_{\alpha}$ and $\pi$ if and only if $P O \pi$ is a 4-space which contains the tangent to $C_{\alpha}$ at one of these intersections. Thus, there is a unique plane $\sigma=P O Q$ which passes through $P$ and $O$, meets $\pi$ (since it lies in an $S_{4}$ with $\pi$ ), and intersects $C_{\alpha}$ in a point distinct (in general) from the $\alpha-1$ intersections of $C_{\alpha}$ and $\pi$. Now $\sigma$ meets $V_{4}^{2}$ in a conic, $\gamma$, and this conic meets the given linear complex $\Gamma$ in two points, say $M$ and $N$. Finally, $P$ (which of course lies on $\gamma$ ) has a unique harmonic con-

Received by the editors November 24, 1952. 
jugate, $P^{\prime}$, with respect to $M$ and $N$ on the conic $\gamma$. We consider the transformation that takes $P$ to $P^{\prime}$; this is obviously involutory. If $P$ is distinct from $M$ and $N$, i.e., if $P$ is not in the linear complex $\Gamma$, then $P$ and $P^{\prime}$ are necessarily distinct, and so $P$ cannot be invariant. On the other hand, if $P$ is in $\Gamma$, then on $\gamma, P$ coincides either with $M$ or with $N$ and, from the elementary properties of harmonic ranges, $P^{\prime}$ must coincide with $P$, i.e., $P$ is an invariant point. Hence, the points of $\Gamma$, and only those points, are invariant. Thus, the involution has a linear complex of invariant elements, as desired, and it remains to determine the order, $m$, of the involution and verify that it can take on any value $\geqq 4$.

2. The order of the involution. To determine $m$ it is convenient to solve first for the number, $k$, of points on a general line $l$ of $V_{4}^{2}$ which are singular, i.e. have the property that the line $P P^{\prime}$ lies entirely on $V_{4}^{2}$. Then we can find $m$ at once from the formula $m=k+2 i-1$ of [1]; in fact, since $i=1$ in the present case, $m=k+1$.

To find $k$ we observe first that the line joining a point $P$ to its image $P^{\prime}$ will lie entirely on $V_{4}^{2}$ if and only if the plane $\sigma$ determined by $P$ meets $V_{4}^{2}$ in a conic consisting of a pair of lines. Moreover, when this is the case, one of the lines must pass through $O$.

Now consider a general line $l$ on $V_{4}^{2}$. From the nature of the involution it is evident that the points of $l$ are in $1: 1$ correspondence with the points of $C_{\alpha}$. On $l$ there are three and only three classes of points for which $\sigma$ meets $V_{4}^{2}$ in a pair of lines. These arise respectively when the line of $V_{4}^{2}$ which passes through $O$ in $\sigma$ :

1. meets $l$,

2. meets $C_{\alpha}$,

3. meets $\pi$ in a point distinct from any of the $\beta$ intersections of $\lambda$ and $C_{\alpha}$.

In Case 1 , the singular point, $L$, on $l$ is unique, being in fact the intersection of $l$ and the $S_{4}$ which is tangent to $V_{4}^{2}$ at $O$.

In Case 2, we note that the $S_{4}$ which is tangent to $V_{4}^{2}$ at $O$ meets $C_{\alpha}$ in $\alpha$ points, consisting of the $\beta$ points $Q_{j}^{(1)}$ common to $C_{\alpha}$ and $\lambda$, and $\alpha-\beta$ additional points, $Q_{j}^{(2)}$, which are not in $\pi$. The line joining each of these points to $O$ obviously lies entirely on $V_{4}^{2}$. Now $\pi$ and the line joining $O$ to any of the $\alpha-\beta$ points $Q_{j}^{(2)}$ determine an $S_{4}$ which meets $l$ in a point, say $L_{j}^{(2)}$. Similarly, $\pi$, the line joining $O$ to any one of the $\beta$ points $Q_{j}^{(1)}$, and the tangent to $C_{\alpha}$ at $Q_{j}^{(1)}$, determine an $S_{4}$ which meets $l$ in a point, say $L_{j}^{(1)}$. Any of the points $L_{j}^{(1)}$ and $L_{j}^{(2)}$ determine with $O$ and the corresponding point $Q_{j}^{(1)}$ or $Q_{j}^{(2)}$ a plane $\sigma$ which passes through $O$, meets $C_{\alpha}$ and $\pi$, and intersects $V_{4}^{2}$ in a com- 
posite conic. Moreover, the $L_{j}^{(1)}$ and $L_{j}^{(2)}$ are clearly all distinct, and different from the point $L$ obtained in Case 1. Thus the $L_{j}^{(1)}$ and $L_{j}^{(2)}$ constitute $\alpha$ additional singular points on $l$.

Finally, in Case 3, there is a unique plane of $V_{4}^{2}$ passing through $O$ and meeting $\pi$ in a line, namely the plane of $O \lambda$. This plane and $l$ determine an $S_{4}$ which meets $C_{\alpha}$ in $\alpha$ points, $R_{j}$, including the $\beta$ intersections of $C_{\alpha}$ and $\lambda$ which we have already taken into account, and hence now reject. In this $S_{4}$, the 3-space $l O R_{j}$ meets $\lambda$ in a point, say $G_{j}$. Moreover, since $l$ and the plane $\sigma=O R_{j} G_{j}$ lie in the same 3-space, $\sigma$ meets $l$ in a point, say $L_{j}$. Since $\sigma$ clearly passes through $O$, meets $l$, $C_{\alpha}$, and $\pi$, and intersects $V_{4}^{2}$ in a composite conic (consisting of the lines $O G_{j}$ and $L_{j} R_{j}$ ) the points $L_{j}$ are also singular points on $l$. Further, it is clear that the $L_{i}$ are all distinct and different from $L$ and any of the points $L_{j}^{(1)}$ and $L_{j}^{(2)}$. Hence they constitute $\alpha-\beta$ additional singular points in the set of singular points on $l$ which we are enumerating.

Therefore, on $l$ we have altogether

$$
k=1+\alpha+(\alpha-\beta)=2 \alpha+\beta+1
$$

singular points. Hence, the order of the involution is

$$
m=k+1=2 \alpha+\beta+2 \quad(\beta \leqq \alpha-1) .
$$

Thus beginning with $\alpha=1$ and $\beta=0$ we can obtain involutions of all orders $\geqq 4$ possessing linear complexes of invariant elements.

\section{REFERENCE}

1. C. R. Wylie, Jr., A new series of line involutions in $S_{3}$, Mathematics Magazine, January-February (1950) pp. 125-131.

The UNIVERSITY OF UTAH 